\title{
Effect of brefeldin A and castanospermine on resistant cell lines as supplements in anticancer therapy
}

\author{
KAROLINA WOJTOWICZ ${ }^{1}$, RADOSŁAW JANUCHOWSKI ${ }^{1}$, PATRYCJA SOSIŃSKA ${ }^{2}$, \\ MICHAŁ NOWICKI ${ }^{1}$ and MACIEJ ZABEL ${ }^{1,3}$ \\ ${ }^{1}$ Department of Histology and Embryology, Poznan University of Medical Sciences, 60-781 Poznan; \\ ${ }^{2}$ Department of Pathophysiology, Poznan University of Medical Sciences, 60-806 Poznań; \\ ${ }^{3}$ Department of Histology and Embryology, Wroclaw Medical University, 50-368 Wrocław, Poland
}

Received November 17, 2015; Accepted December 17, 2015

DOI: $10.3892 / o r .2016 .4656$

\begin{abstract}
In the present study, we analyzed the influence of brefeldin A (BFA) and castanospermine (CAS) on the activity, stability and localization of P-glycoprotein (Pgp) and breast cancer resistance protein (BCRP) in various resistant cell lines. The impact of BFA and CAS on cell viability was assessed using the MTT test. Western blotting (WB) was performed to assess the effect of the inhibitors on the expression of the investigated proteins. Immunofluorescence was employed to assess the effect of BFA and CAS on the cellular localization of the proteins. Flow cytometry was used to verify the functional role of inhibitors on drug uptake and efflux. The MTT test showed that BFA had a significant effect on cell viability in LoVo/Dx and W1PR cell lines. WB analysis demonstrated that BFA partially blocked Pgp $N$-glycosylation and induced BCRP degradation and CASP 3-dependent apoptosis in W1TR cells; however, the BFA activity was p53-independent. CAS had no effect on the stability of Pgp but increased the level of non-glycosylated BCRP. The expression of p53 protein decreased in all of the cells that were treated with CAS. Immunofluorescence revealed that BFA caused a more granular Pgp signal in W1PR and BCRP in A2780T1 cells. Furthermore, BFA caused morphological changes in LoVo/ Dx and W1TR cell lines. CAS also induced a granular signal
\end{abstract}

Correspondence to: Karolina Wojtowicz, Department of Histology and Embryology, Poznan University of Medical Sciences, 6 Święcickiego Street, 60-781 Poznań, Poland

E-mail: k_wojtowicz@onet.pl

Abbreviations: BFA, brefeldin A; CAS, castanospermine; ABC, ATP-binding cassette superfamily; BCRP/ABCG2, breast cancer resistance protein; BSA, bovine serum albumin; CASP 3, caspase 3; FBS, fetal bovine serum; GAPDH, glyceraldehyde-3-phosphate dehydrogenase; MDR, multidrug resistance; EMEM, Eagle's Minimum Essential Medium; MFI, mean fluorescence intensity; PBS, phosphate-buffered saline; Pgp, glycoprotein P; PNGase F, $\mathrm{N}$-glycosidase F; TBS, Tris-buffered saline

Key words: multidrug resistance, brefeldin A, castanospermine, chemotherapy, glycoprotein P, BCRP in all of the cell lines, except W1TR. The flow cytometry showed higher dye accumulation in sensitive cell lines. We observed an increase in the mean fluorescence intensity (MFI) of Rho123 in LoVo/Dx cells treated with BFA and CAS, but no differences were observed in W1PR. BFA had no effect on the MFI of W1TR, but CAS led to an increase in the level of intracellular H33342 in W1TR and A2780T1 cells. These results suggest that these compounds are likely to be useful as supplements in anticancer therapy.

\section{Introduction}

Although our understanding of cancer has improved compared with previous years, effective treatment is still hampered by the highly complex nature of the disease (1). Chemotherapy is usually the primary treatment for most patients, but multidrug resistance (MDR) often causes chemotherapy failure (2). ATP-binding cassette (ABC) transporters have been found to be involved in MDR. The most important ABC proteins are $\mathrm{P}$-glycoprotein (Pgp) and breast cancer resistance protein (BCRP) $(3,4)$, both of which are glycoproteins and can be affected by glycosylation in MDR (5).

Pgp acts as an ATP-dependent drug efflux pump (6), and its role in MDR was described in 1987 by Pastan and Gottesman (7). Human Pgp contains 10 consensus sequences for $N$-linked glycosylation, but only three of these sequences are glycosylated in the first extracellular loop of Pgp (8). Pgp is synthesized as a $160 \mathrm{kDa}$ protein (8) and is located at the plasma membrane and in intercellular compartments (9-11). BCRP, the other drug transporter protein, is also implicated in the development of the MDR phenotype and has been reported to be overexpressed in many solid tumors (12). It has a molecular mass of $72 \mathrm{kDa}$ and is termed a half-transporter (13) since dimerization is required for substrate transport. The BCRP $N$-linked glycosylation sites correspond to Asn596 in the extracellular loop $(14,15)$.

Glycosylation has been described as the most common post-translational modification of proteins (16). It is involved in various cellular processes, including protein folding, protein secretion, intracellular trafficking, stability and substrate specificity $(15,17)$. Properly folded proteins are secreted or targeted to their final intracellular or extracellular 
destination, whereas misfolded proteins are recognized as aberrant products and targeted for endoplasmic reticulum (ER)-associated degradation (ERAD) $(18,19)$ or delivered to lysosomes for degradation (16).

Glycosylation can be inhibited by different inhibitors that are capable of blocking different stages of this process. The most important inhibitor is tunicamycin, which is used to block the glycosylation of Pgp and BCRP. Other important inhibitors of glycosylation are brefeldin A (BFA) and castanospermine (CAS) (8).

BFA is characterized as an antiviral antibiotic (20). In primary cultured rat hepatocytes, BFA inhibits the secretion of plasma proteins at an early step in the secretory pathway $(21,22)$, as well as the anterograde movement of the membrane beyond the mixed ER/Golgi system (20). Investigators have observed the breakdown of the Golgi apparatus and redistribution of proteins into the ER in the presence of BFA, and these effects were rapidly and completely reversed by removal of the drug (20). BFA treatment of the Golgi apparatus resulted in the rapid induction of membrane tubules (20) and the redistribution of many Golgi enzymes to the ER (23) due to the inhibition of coat protein ( $\beta$-COP) assembly on Golgi cisternae (24). In summary, BFA has been shown to inhibit the exocytotic pathway of proteins from the ER to the Golgi, irrespective of whether these proteins are secretory or membrane-bound, but the effect of the BFA blockade is considered to be only temporary (21). Furthermore, BFA is a potent inducer of apoptosis in human cancer cells based on DNA fragmentation (25), and it induces apoptotic cell death in ovarian carcinoma cell lines by activating the mitochondrial pathway and the caspase- 8 and Bid-dependent pathway (26-28) but not in a p53-dependent mechanism $(25,29)$. Additionally, BFA has been shown to reverse the function of the multidrug resistance-associated protein (MRP) function (30). Although the cytotoxic activity of BFA has been reported, BFA has not yet been applied as an anticancer drug.

CAS is an indolizidine alkaloid that was isolated from the Australian rainforest plant Castanospermum australe (31). The main function of CAS is inhibition of glucosidases I and II and obligatory enzyme trimming during the synthesis of $N$-linked oligosaccharides on glycoproteins (32). In various studies, the ability of CAS to prevent the rejection of organ transplants has been evaluated (33), and it has been shown to display antiviral activity against retroviruses including HIV $(34,35)$. Combination therapy of 6- $O$-butanoyl castanospermine (Celgosivir) with peginterferon and/or ribavirin has been undergoing investigation in phase II clinical trials for the treatment of patients with chronic HCV (36). CAS significantly inhibits tumor growth in nude mice, reduces the adhesion of tumor cells to the vascular endothelium, and decreases antimetastatic activity by inhibiting platelet aggregation of metastatic cells (32). Furthermore, CAS has been shown to inhibit cellular transformation by altering oncogene glycosylation, angiogenesis in drug-treated animals (32) and concentration-dependent inhibition of C6 glioblastoma cell proliferation (37).

Unfortunately, to date, no studies have been conducted to examine the correlation between the changes in MDR protein glycosylation and cancer MDR. Previously, we tested tunicamycin (tun), one of the most well-known inhibitors of glycosylation (38). Research was very encouraging, and therefore we decided to expand our investigation. In the present study, we analyzed BFA and CAS, which block different stages of the glycosylation process. We decided to test the impact of the two $\mathrm{N}$-glycosylation inhibitors on Pgp and BCRP, two of the most common MDR proteins, the tumor overexpression of which is the main cause of cancer chemotherapy failure.

\section{Materials and methods}

Chemicals and drugs. Doxorubicin, topotecan, paclitaxel, BFA, CAS, BMA, MG132, Rho123, H33342 and RIPA lysis buffer were obtained from Sigma (St. Louis, MO, USA). RPMI-1640 medium and Eagle's Minimum Essential Medium (EMEM), fetal bovine serum (FBS), antibiotic-antimycotic solution and L-glutamine were also purchased from Sigma. PNGase F was obtained from New England Biolabs (Hitchin, UK). Bradford dye reagent was obtained from Bio-Rad Laboratories (Hemel Hempstead,UK). Polyvinylidene difluoride (PVDF) membrane was obtained from GE Healthcare (Buckinghamshire, UK). Cell Proliferation Kit I (MTT) and protease inhibitor cocktail were purchased from Roche Diagnostics GmbH (Mannheim, Germany). The rabbit anti-ABCG2 polyclonal Ab (H-70) used for western blotting (WB), rabbit anti-GADPH polyclonal antibody (Ab) (FL-335), goat anti-mouse HRP-conjugated Ab and goat anti-rabbit HRP-conjugated $\mathrm{Ab}$ were purchased from Santa Cruz Biotechnology (Santa Cruz, CA, USA). Mouse monoclonal anti-Pgp Ab (C219) was obtained from Alexis Biochemicals (Lörrach, Germany). The mouse monoclonal anti-p53 $\mathrm{Ab}$ and mouse monoclonal anti-ABCG2 used for the immunofluorescence experiments were obtained from Novus Biologicals (Germany). Human/mouse cleaved caspase-3 (Asp175) monoclonal antibody (MAB835) was obtained from R\&D Systems (Abingdon, UK). The MFP488 fluorescent secondary antibodies was obtained from MoBiTec (Goettingen, Germany). Mounting medium with DAPI was obtained from Santa Cruz Biotechnology. The 4X Laemmli sample buffer and 4-20\% mini-PROTEAN ${ }^{\circledR}$ TGX $^{\mathrm{TM}}$ precast gels were obtained from Bio-Rad Laboratories.

Cell culture. Two resistant ovarian cancer cell lines were used in the present study. From the W1 cell line \{primary ovarian cancer cell line, established from ovarian cancer tissues that were obtained from an untreated patient in December 2009 [these cell lines were derived in our laboratory as described previously by Januchowski et al (39)]\}, we generated the W1TR subline, which is resistant to topotecan (top) (W1 is topotecan resistant to a top concentration of $24 \mathrm{ng} / \mathrm{ml}$ ), and the W1PR subline, which is resistant to paclitaxel (pac) (W1 is paclitaxel resistant to a pac concentration of $1,100 \mathrm{ng} / \mathrm{ml}$ ). The second ovarian cancer cell line, A2780T1, is a topotecan-resistant subline that was generated from the commercially available established human ovarian carcinoma cell line A2780 (obtained from ATCC, Poland). The drug-resistant sublines were generated by the exposure of the drug-sensitive cells to incrementally higher concentrations of each drug. The human colon adenocarcinoma doxorubicin (dox)-resistant cell line LoVo and LoVo/Dx were also used (obtained from ATCC). LoVo/Dx was cultured in the presence of $200 \mathrm{ng} / \mathrm{ml}$ dox to maintain its resistant phenotype. 
The LoVo, LoVo/Dx, A2780 and A2780T1 cell lines were cultured in EMEM; and the W1, W1PR and W1TR cell lines were cultured in RPMI-1640 medium. The media were supplemented with 10\% FBS, 2 mM L-glutamine and $1 \%$ antibiotic-antimycotic solution. The cells were cultured at $37^{\circ} \mathrm{C}$ in a humidified atmosphere with $5 \% \mathrm{CO}_{2}(\mathrm{v} / \mathrm{v})$.

The W1TR and A2780TR1 cells were shown to overexpress BCRP, while the W1PR and LoVo/Dx cells overexpress Pgp $(39,40)$.

Cell viability assay. To determine the cell viability we used the Cell Proliferation Kit I (MTT) according to the manufacturer's instructions. The survival assay was performed to estimate the extent of cell resistance to the chemotherapeutic agents, BFA and CAS. Briefly, the cells were seeded at $4 \times 10^{3}$ cells/well $(200 \mu \mathrm{l})$ in 96-well culture plates and pre-incubated for $48 \mathrm{~h}$. To examine the effect of BFA, CAS or chemotherapeutic drugs on cell survival, the cells were treated with increasing concentrations of BFA, CAS or the different drugs for $72 \mathrm{~h}$. Subsequently, $10 \mu \mathrm{l}$ of MTT labeling reagent was added to the medium (the final concentration of MTT was $0.5 \mathrm{mg} / \mathrm{ml}$ ) for $4 \mathrm{~h}$ and $100 \mu \mathrm{l}$ of the solubilized solution was then added to each well. After an overnight incubation, the absorbance was measured in a microplate reader at $570 \mathrm{~nm}$ with a reference wavelength of $720 \mathrm{~nm}$.

In addition, we examined the effect of BFA and CAS on cellular resistance to chemotherapeutic drugs. The cells were seeded at $4 \times 10^{3}$ cells/well in 96-well culture plates. After $48 \mathrm{~h}$, the cells were pre-treated with BFA or CAS for another $48 \mathrm{~h}$, and the medium was subsequently replaced with fresh medium supplemented with a cytostatic drug and BFA or CAS. The BFA concentration used was based on previously established values for each cell line: $10 \mathrm{ng} / \mathrm{ml}$ for LoVo/ Dx, $7 \mathrm{ng} / \mathrm{ml}$ for W1PR, $6 \mathrm{ng} / \mathrm{ml}$ for W1TR and $8 \mathrm{ng} / \mathrm{ml}$ for A2780T1 cells; for CAS: $10 \mu \mathrm{g} / \mathrm{ml}$ for LoVo/Dx, $50 \mu \mathrm{g} / \mathrm{ml}$ for W1PR and W1TR and $60 \mu \mathrm{g} / \mathrm{ml}$ for A2780T1 cells. After $72 \mathrm{~h}$ of incubation, cell proliferation was assessed as previously described. As a control, we used cells that were treated with only the cytostatic drugs for $72 \mathrm{~h}$. All experiments were repeated three times.

Preparation of cell lysates and glycosidase treatment. For the immunoblot analysis of protein expression, the cells were pre-incubated for $48 \mathrm{~h}\left(1 \times 10^{6}\right.$ cells $/ 75 \mathrm{~cm}^{2}$ culture flask). The medium was then replaced with fresh medium only or fresh medium supplemented with BFA or CAS at different concentrations. After $72 \mathrm{~h}$ of incubation, the cells were harvested by trypsinization and pelleted by centrifugation. The cell pellets were washed once with phosphate-buffered saline (PBS), and the cells were lysed with RIPA lysis buffer supplemented with protease inhibitor cocktail for $5 \mathrm{~min}$ on ice. The cell lysates were centrifuged at $8,000 \mathrm{xg}$ at $4^{\circ} \mathrm{C}$ for $10 \mathrm{~min}$, and the total protein concentration in the supernatant was measured. The protein concentration was determined using Bradford dye reagent with BSA as the standard. Glycosidase treatment was performed by incubating $35 \mu \mathrm{g}$ of the cell lysate with $5 \mu \mathrm{l}$ of PNGase $\mathrm{F}$ at $37^{\circ} \mathrm{C}$ for $10 \mathrm{~min}$.

Western blot analysis. Western blot analysis was conducted under reducing conditions. The cell lysate samples $(35 \mu \mathrm{g})$ were initially mixed with $4 \mathrm{X}$ Laemmli sample buffer and incubated at room temperature (RT) for $20 \mathrm{~min}$. The lysates were then electrophoretically separated on a $4-20 \%$ miniPROTEAN ${ }^{\circledR}$ TGX $^{\mathrm{TM}}$ precast gel and then electroblotted onto a PVDF membrane. The membrane was blocked with 5\% (w/v) dry milk in TBS at RT for $1 \mathrm{~h}$. The membranes were then incubated with anti-Pgp, anti-BCRP, anti-p53 primary (1:500 dilution) and anti-CAS 3 (1:1,000 dilution) antibodies at $4^{\circ} \mathrm{C}$ overnight. The membranes were also probed with the anti-GAPDH antibody (1:1,000 dilution) as a loading control. For the secondary antibody, horseradish peroxidase (HRP)-linked anti-species antibody was used at a dilution of 1:2,000. HRP-dependent luminescence was developed with the Femto SuperSignal reagent and detected using a UVP imaging system.

Immunofluorescence analysis. Cells were fixed and permeabilized in $4 \%$ paraformaldehyde for $15 \mathrm{~min}$, followed by ice-cold methanol for $15 \mathrm{~min}$ at $-20^{\circ} \mathrm{C}$ and washed with PBS. The cells were then incubated with 5\% HSA for $1 \mathrm{~h}$. To detect Pgp, the cells were treated with a mouse monoclonal antibody against Pgp at a dilution of 1:30 for $1 \mathrm{~h}$ at RT. The cells were then washed five times with PBS and incubated with an MFP488-labeled anti-mouse antibody for $1 \mathrm{~h}$ at RT in the dark. To visualize the cell nuclei, the cells were mounted with a DAPI-containing mounting medium (blue). To detect BCRP, the cells were treated with a mouse monoclonal anti-BCRP antibody (Novus Biologicals) at a dilution of 1:200 for $1 \mathrm{~h}$ at RT. Subsequently, the cells were washed with PBS and incubated with an MFP488-labeled anti-mouse secondary antibody. Finally, the coverslips were mounted with DAPI mounting medium. Fluorescent images were collected using a ZEISS light microscope. The expression of Pgp and BCRP was analyzed by pseudo-color representations of the fluorescence intensity (green).

Flow cytometric analysis. To assess the efflux activity of Pgp and BCRP, whole resistant cell lines were incubated with BFA or CAS and drugs, using previously described conditions. We used sensitive W1, A2780 and LoVo cell lines as a control for the inability to eliminate the drugs from cells. In addition, W1PR, W1TR, A2780T1 and LoVo/Dx cells were used as a positive control for drug efflux. We used the fluorescent dye Rhodamine 123 (Rho123) as an index of Pgp activity and Hoechst33342 (H33342) as an index of BCRP activity. The cell suspensions $\left(1 \times 10^{6} / \mathrm{ml}\right)$ were incubated with $0.5 \mu \mathrm{g} / \mathrm{ml} \mathrm{Rho123}$ or $2.5 \mu \mathrm{g} / \mathrm{ml} \mathrm{H} 33342$ for $1 \mathrm{~h}$ at $37^{\circ} \mathrm{C}$ in medium. Next, the cells were washed twice in cold PBS with $500 \mu \mathrm{M}$ verapamil (a known MDR inhibitor), and cellular uptake of Rho123 or H33342 was immediately analyzed using a FACSAria III (BD, Warsaw, Poland) with the FCS Express Plus software program. In each analysis, 10,000 events were recorded. The fluorescent emission was collected at $488 \mathrm{~nm}$ for Rho1 23 and at $375 \mathrm{~nm}$ for $\mathrm{H} 33342$.

Statistical analysis. The statistical analysis was performed using Microsoft Excel software. The statistical significance of the differences was determined by applying the Student's t-test. A p-value $\leq 0.05$ was considered to indicate a statistically significant result. 

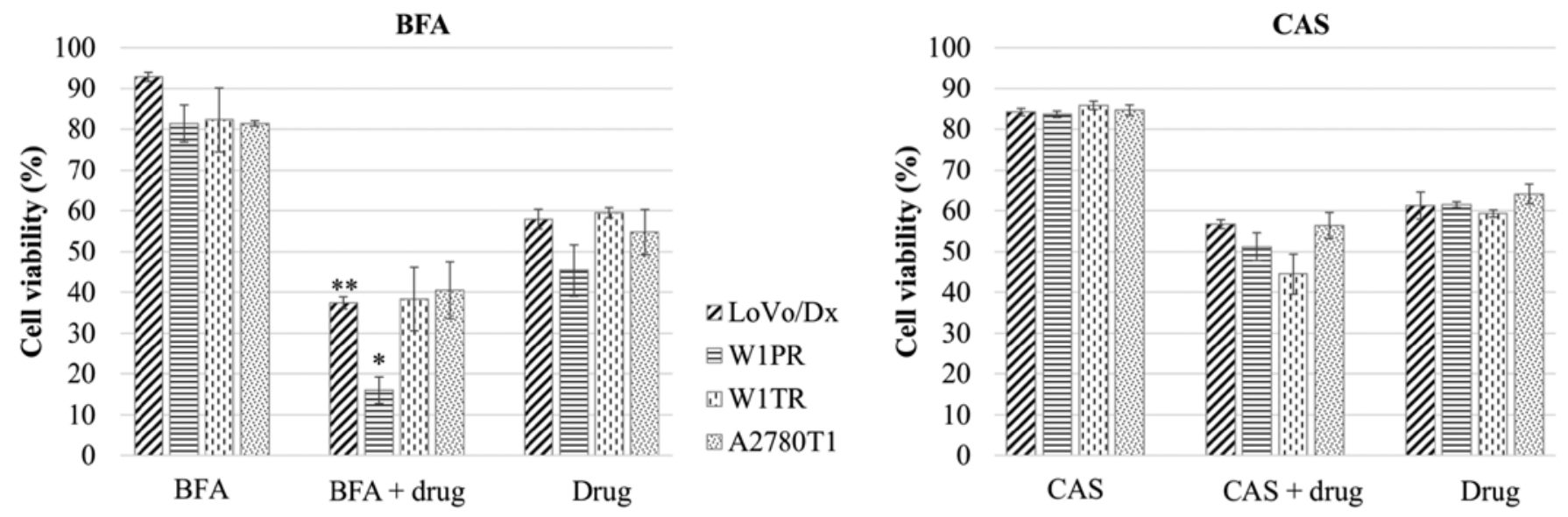

Figure 1. MTT cell survival assay. LoVo/Dx, W1PR, W1TR and A2780T1 cells were seeded at a density of 4,000 cells/well in 96-well plates. The cells were pre-treated with BFA or CAS for $48 \mathrm{~h}$, and then with a combination of BFA or CAS and doxorubicin (dox) (LoVo/Dx), topotecan (top) (W1TR and A2780T1), or paclitaxel (pac) (W1PR) for $72 \mathrm{~h}$. The cell viability of each cell line is expressed as a percentage of the untreated control. Data are expressed as mean values \pm SD from three independent experiments, ${ }^{* *} \mathrm{p}<0.01,{ }^{*} \mathrm{p}<0.05$.

\section{Results}

Effect of BFA and CAS on cell viability. To identify the impact of BFA and CAS on cell viability, the cells were treated with BFA or CAS at increasing concentrations for $72 \mathrm{~h}$. BFA and CAS induced a dose-dependent decrease in cell viability in all of the drug-resistant cell lines (data not shown). In subsequent experiments, we selected an inhibitor concentration that would only decrease cell viability to $81-93 \%$ in each cell line (Fig. 1). We decided to use an inhibitor concentration that was not too toxic to the cells, but that provided an observable effect. To achieve this goal, BFA was applied at a concentration of $10 \mathrm{ng} /$ $\mathrm{ml}$ for LoVo/Dx, $7 \mathrm{ng} / \mathrm{ml}$ for W1PR, $6 \mathrm{ng} / \mathrm{ml}$ for W1TR and $8 \mathrm{ng} / \mathrm{ml}$ for A2780T1 cells, and CAS was used at a concentration of $10 \mu \mathrm{g} / \mathrm{ml}$ for LoVo/Dx, $50 \mu \mathrm{g} / \mathrm{ml}$ for W1PR and W1TR and $60 \mu \mathrm{g} / \mathrm{ml}$ for A2780T1 cells.

Next, we tested whether BFA or CAS and the cytostatic drugs had a synergistic effect on the drug-resistant cell lines. Cells were treated with BFA or CAS at the indicated concentrations for $48 \mathrm{~h}$. The medium was then replaced with fresh medium supplemented with BFA or CAS and one of the cytostatic drugs at the following concentrations: dox $200 \mathrm{ng} / \mathrm{ml}$, top $24 \mathrm{ng} / \mathrm{ml}$ and pac $1,100 \mathrm{ng} / \mathrm{ml}$. The cells were incubated for another $72 \mathrm{~h}$. We also treated cells with the cytostatic drugs alone for $72 \mathrm{~h}$. As a control, cells were assessed in the absence of cytostatic drugs.

In the LoVo/Dx and W1PR cell lines, we observed a statistically significant synergistic effect of BFA and the drugs (Fig. 1). Furthermore, the viability of the LoVo/Dx cell line in response to the co-treatment with BFA and dox showed the most significant decrease $(\mathrm{p}<0.01)$ in comparison to the cells that were treated with dox. Co-treatment of the W1TR cell line with BFA and top resulted in reduced cell viability in comparison to cells that were treated with top alone, but the changes were not statistically significant $(\mathrm{p}<0.1)$. In the A2780T1 cells supplemented with BFA and top, there were no significant changes in viability in comparison to cells that were treated with top alone (Fig. 1).

CAS treatment had no statistically significant effect on the sensitivity of tumor cells to cytostatic drugs (Fig. 1), although in W1PR, W1TR and A2790T1 cells, CAS reduced the cell viability in comparison to cells that were treated with the drug alone, but the changes were not statistically significant $(\mathrm{p}<0.1)$.

\section{Effect of BFA and CAS on the expression of Pgp and BCRP} proteins. Western blot analyses were conducted to assess whether BFA or CAS could affect the expression of Pgp and BCRP proteins. After BFA treatment, Pgp displayed a molecular mass that was approximately a few kDa less than that of the fully glycosylated protein (Fig. 2A). As a control, the LoVo/Dx and W1PR cell lysates were incubated with $N$-glycosidase F (PNGase F) to remove all of the $N$-linked glycans. After incubation with PNGase F, we observed bands at an even lower molecular weight then those observed for the BFA-treated cells. We did not observe any changes in the level of p53 protein expression in the LoVo/Dx or W1PR cells after BFA treatment (Fig. 2A).

For BCRP, it was characteristic to identify two bands in the control samples, indicating two forms of the BCRP protein (Figs. 2A and 3). After incubation with PNGase F, only the band with the lower molecular weight was observed (Fig. 2A). The lower band was consistent with the size of the unglycosylated protein. In the BFA-treated cells, we observed a third, additional band (Fig. 2A), and a more fully glycosylated form of BCRP. We did not notice any changes in p53 expression in the A2780T1 cells following treatment with BFA, in contrast to W1TR cells in which the level of p53 protein was reduced after BFA supplementation (Fig. 2A). BFA treatment increased and decreased the expression of CASP 3 in the W1TR and W1PR cell lines, respectively (Fig. 2B).

Due to the observation of a third band of BCRP in BFA-treated A2780TR1 and W1TR cells, we decided to further investigate the mechanism underlying this phenomenon. We suspect that BFA could affect BCRP degradation. Protein degradation occurs at two major sites: the lysosome and the proteasome. To examine whether the lysosomal or the ubiquitin-proteasomal pathway was involved in BCRP degradation, we pre-cultured A2780TR1 and W1TR cells in the presence or absence of $2 \mu \mathrm{M}$ MG132 (a proteasomal degradation inhibitor) or $10 \mathrm{nM}$ BMA (a lysosomal degradation 
A

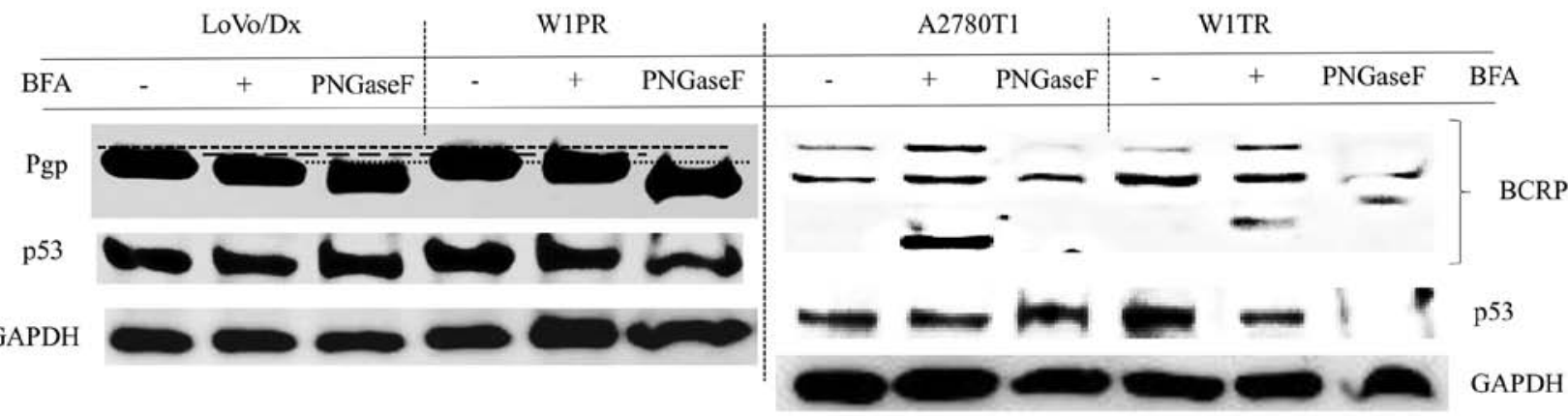

B$$
\text { BFA }
$$ LoVo/Dx BFA

CASP 3

GAPDH

\begin{tabular}{l|l|l}
$\mathrm{LoVo} / \mathrm{Dx}$ & $\mathrm{W} 1 \mathrm{P}$ \\
- & + & - \\
\hline & &
\end{tabular}

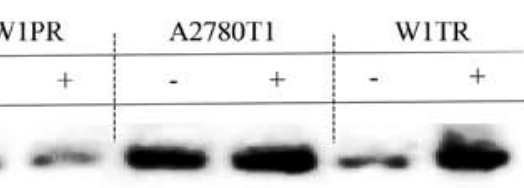

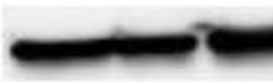

C

\begin{tabular}{ccc|crr}
\multicolumn{2}{c|}{ A2780T1 } & \multicolumn{3}{c}{ W1TR } \\
\hline BFA & BFA & BFA & BFA & BFA & BFA \\
& BMA & MG132 & & BMA & MG132 \\
\hline
\end{tabular}

BCRP

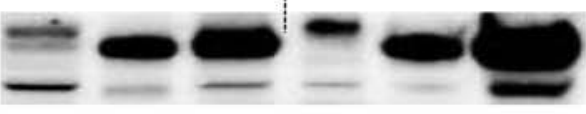

GAPDH

Figure 2. Effect of brefeldin A (BFA) on the expression of Pgp, BCRP, p53 and CASP 3 proteins in LoVo/Dx, W1PR, A2780T1 and W1TR cell lines. As a control, protein lysate was digested with PNGase F. Cellular proteins $(35 \mu \mathrm{g})$ were separated on a $4-20 \%$ mini-PROTEAN ${ }^{\circledR}$ TGX $^{\mathrm{TM}}$ precast gel and transferred to a PVDF membrane. The membrane was blocked and immunoblotted with a primary Ab and then an HRP-conjugated secondary Ab. (A) Pgp, BCRP and p53 expression in BFA-treated cells and after PNGase F digestion. (B) CASP 3 expression in all BFA-treated cells. (C) The effect of BFA and either BMA or MG132 on BCRP expression in the A2780T1 and W1TR cells. GAPDH was used as a loading control for the western blot analyses.

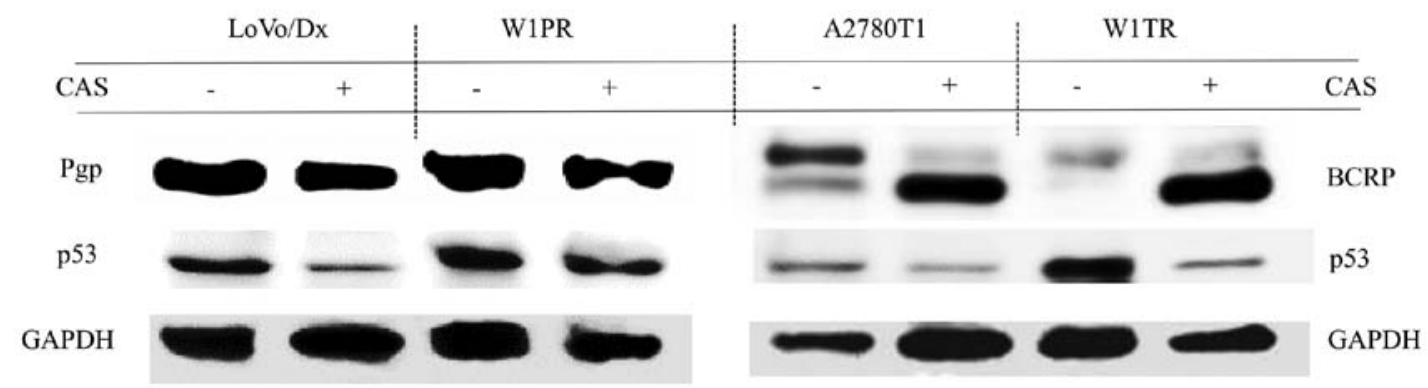

Figure 3. Effect of castanospermine (CAS) on the protein expression of Pgp, BCRP and p53 in LoVo/Dx, W1PR, A2780T1 and W1TR cell lines. Cellular proteins $(35 \mu \mathrm{g})$ were separated on a 4-20\% mini-PROTEAN ${ }^{\circledR}$ TGX $^{\mathrm{TM}}$ precast gel and transferred to a PVDF membrane. The membrane was blocked and immunoblotted with a primary Ab and then an HRP-conjugated secondary Ab. GAPDH was used as a loading control for the western blot analyses.

inhibitor) for $3 \mathrm{~h}$ and subsequently replaced the medium with fresh medium supplemented with BFA and MG132 or BMA for another $72 \mathrm{~h}$. We verified that the concentrations of the protein degradation inhibitors were non-toxic to the cells. We found that pre-treatment with MG132 or BMA led to an increase in the expression only of unglycosylated BCRP, but the lowest band was still observed (Fig. 2C). Moreover, BCRP protein expression was increased in the W1TR cells that were co-treated with BFA and MG132, despite the lower total protein concentration represented by GAPDH (Fig. 2C).

Pgp bands of the same size were obtained from $\mathrm{LoV} / \mathrm{Dx}$ and W1PR cells treated with CAS compared with the controls without drug (Fig. 3). BCRP was detected in the form of two bands in the A2780T1 and W1TR cell lines. The upper bands corresponding to the fully glycosylated form of BCRP were more apparent. In cells treated with CAS, BCRP was mainly detected as a lower-molecular-weight protein (Fig. 3) corresponding to the size of unglycosylated BCRP. Reduced expression of $\mathrm{p} 53$ was detected in all of the cells treated with CAS (Fig. 3).
Effect of BFA and CAS on the cellular localization of Pgp and $B C R P$. We were interested in whether BFA or CAS affected the cellular localization of Pgp in W1PR and LoVo/Dx cells and the localization of BCRP in W1TR and A2780T1 cells. In LoVo/Dx cells, Pgp was mostly detected at the plasma membrane, in the cytoplasm and around the nuclear envelope in the absence of BFA or CAS treatment (Fig. 4). After BFA treatment, we noticed changes in the morphology of the cells; they became rounder, while the Pgp signal remained the same as in the control. In contrast, in CAS-treated cells, Pgp was mainly observed within the cytoplasm in the form of granules (Fig. 4). In W1PR cells, the localization of Pgp was similar to that in the LoVo/Dx cells; it was observed at the plasma membrane, in the cytoplasm and close to the nuclear envelope. BFA treatment caused a Pgp signal in the cytoplasm to move to the nuclear periphery. In the CAS W1PR cells, the fluorescence signal was mostly cytoplasmic, which was a little different compared with the other cells (Fig. 4).

In the untreated A2780T1 and W1TR cells, BCRP was detected in the cytoplasm (Fig. 4). After BFA treatment, the 


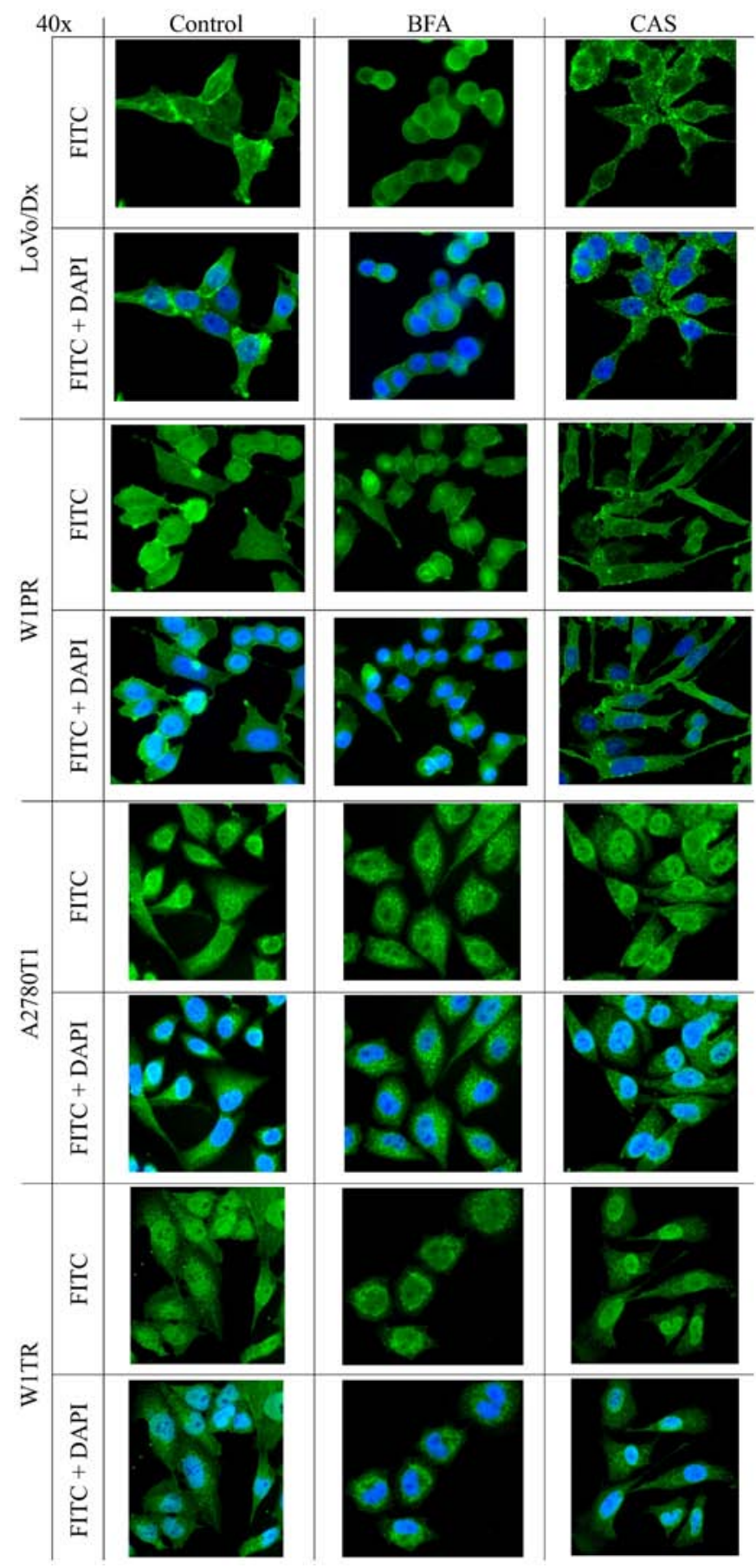

Figure 4. Immunofluorescence analysis of Pgp and BCRP expression in LoVo/Dx, W1PR, W1TR and A2780T1 cell lines in the absence or presence of brefeldin A (BFA) and castanospermine (CAS). Pgp was detected using anti-Pgp and MFP488-conjugated secondary antibodies (green). BCRP was detected using an anti-BCRP antibody (Novus Biologicals) and an MFP488-conjugated secondary antibody (green). To visualize cell nuclei, the cells were mounted with a DAPI-containing mounting medium (blue). Magnification, $\mathrm{x} 40$.

localization of BCRP in the A2780T1 cell line changed to a granular signal. The morphology of W1TR cells changed after BFA treatment, exhibiting a rounder appearance compared with the control (Fig. 4). CAS treatment affected BCRP localization only in A2780T1 cells, in which BCRP protein aggregates were observed in the cytoplasm (Fig. 4).
Effect of BFA and CAS on the cellular accumulation of Rho123 or H33342. To verify whether BFA or CAS could have a functional role in drug uptake and efflux, fluorescence accumulation and efflux were investigated in the absence and presence of BFA or CAS. The intracellular uptake of the fluorescent dye Rho123 or H33342 was evaluated in sensitive and MDR cell lines, and in MDR cells treated with BFA or CAS. A precise analysis was conducted based on the mean fluorescence intensity (MFI). The results indicated that the amount of Rho123 or H33342 accumulation in sensitive cells was increased compared with that in resistant cells in all cell lines (Fig. 5). The level of intracellular Rho123 was 2.7 times higher in LoVo than that in the LoVo/Dx cells and 20 times higher in W1 than that in the W1PR cells (Fig. 6). Similarly, the intracellular level of H33342 was 2.2 times higher in W1 than that in the W1TR cells and 5 times higher in A2780 than that in the A2780T1 cells (Fig. 6). We observed an increase in the MFI of Rho123 in the LoVo/Dx cells treated with BFA and CAS in comparison with the LoVo/Dx control sample, but these changes were not significant (Figs. 5 and 6). No differences in the MFI of Rho123 were detected in the W1PR cells after BFA and CAS stimulation. The addition of BFA to W1TR cells had no effect on MFI, but CAS led to a significant increase in the level of intracellular $\mathrm{H} 33342$ in the resistant W1TR cells (Figs. 5 and 6). The analogous effect was observed in A2780T1 cells, in which CAS increased the MFI of H33342 in comparison to BFA (Figs. 5 and 6).

\section{Discussion}

The expression of MDR proteins, including Pgp and BCRP, is responsible for chemotherapy failure in numerous cancers. Consequently, the application of drugs that block the activity and function of MDR proteins represents an opportunity in current research in oncology. New strategies are needed to circumvent the effects of MDR in cancer chemotherapy. Since both proteins are glycosylated, it can be anticipated that the use of glycosylation inhibitors will change their expression and increase the sensitivity of cells to cytostatic drugs.

In the present study, we tested two inhibitors of $\mathrm{N}$-linked glycosylation, BFA and CAS, as potential factors facilitating an increase in the efficiency of chemotherapy. BFA inhibits protein transport from the ER to the Golgi apparatus (20). In the present study, BFA increased sensitivity to cytostatic drugs in LoVo/Dx and W1PR cell lines characterized by Pgp overexpression. This finding is consistent with the results of $\mathrm{Fu}$ et al, who also showed that BFA increased the intracellular accumulation of the Pgp substrate daunorubicin, resulting in a higher toxicity in HeLa cells (41). Fu et al stated that this phenomenon resulted from an increase in the localization of Pgp in the ER (41). We suspected that BFA had a similar effect on Pgp in the present analyses. BFA also significantly increased the intercellular accumulation of ZDV, another Pgp substrate, in a pre-adipocyte cell line (42). We suggest that BFA induces an increase in the accumulation of chemotherapeutic agents in the cytoplasm of cells that are characterized by Pgp overexpression. Furthermore, Rhodes et al demonstrated that BFA blocks non-Pgp-mediated MDR activity in the COR-L23/R cell line (43). In contrast, other researchers reported that BFA has no effect on the cellular drug level (44-46). Therefore, we 


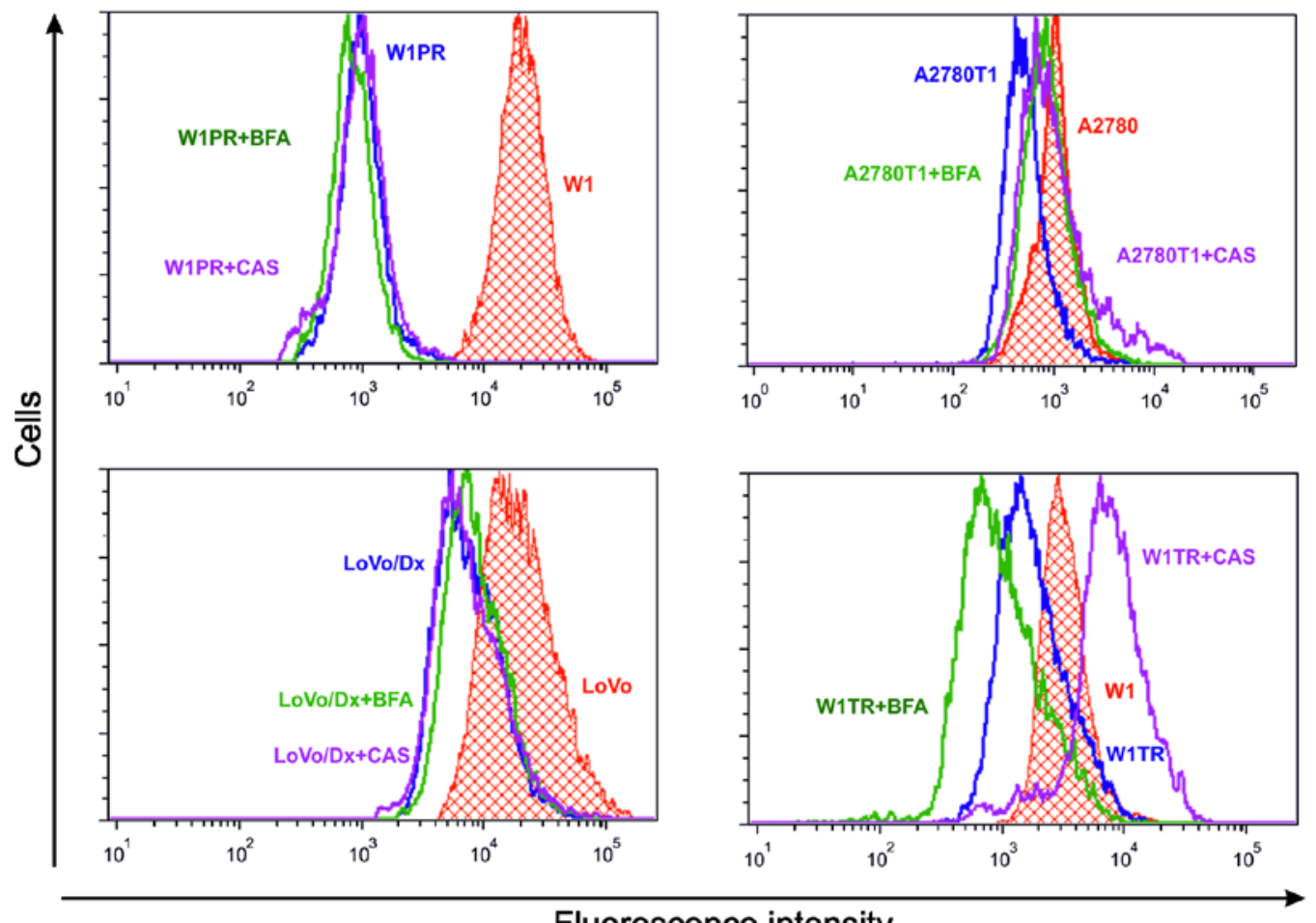

Fluorescence intensity

Figure 5. The influence of BFA and CAS on intercellular Rho123 and H33342 accumulation in the tested cell lines. Cells were exposed to $0.5 \mu \mathrm{g}$ Rho123/ml in medium or $2.5 \mu \mathrm{g} \mathrm{H} 33342 / \mathrm{ml}$ in medium at $37^{\circ} \mathrm{C}$ for $1 \mathrm{~h}$ and then washed twice with cold PBS containing $500 \mu \mathrm{M}$ verapamil to inhibit MDR activity. The figure shows four diagrams of the fluorescence intensity, each consisting of the fluorescence intensity of the sensitive cell line, corresponding resistant cell line and resistant cells treated with the inhibitors. Whole control sensitive cell lines that were not treated with inhibitors are indicated in red, untreated resistant cells are indicated in blue, and cells treated with BFA and CAS are indicated in green and purple, respectively.

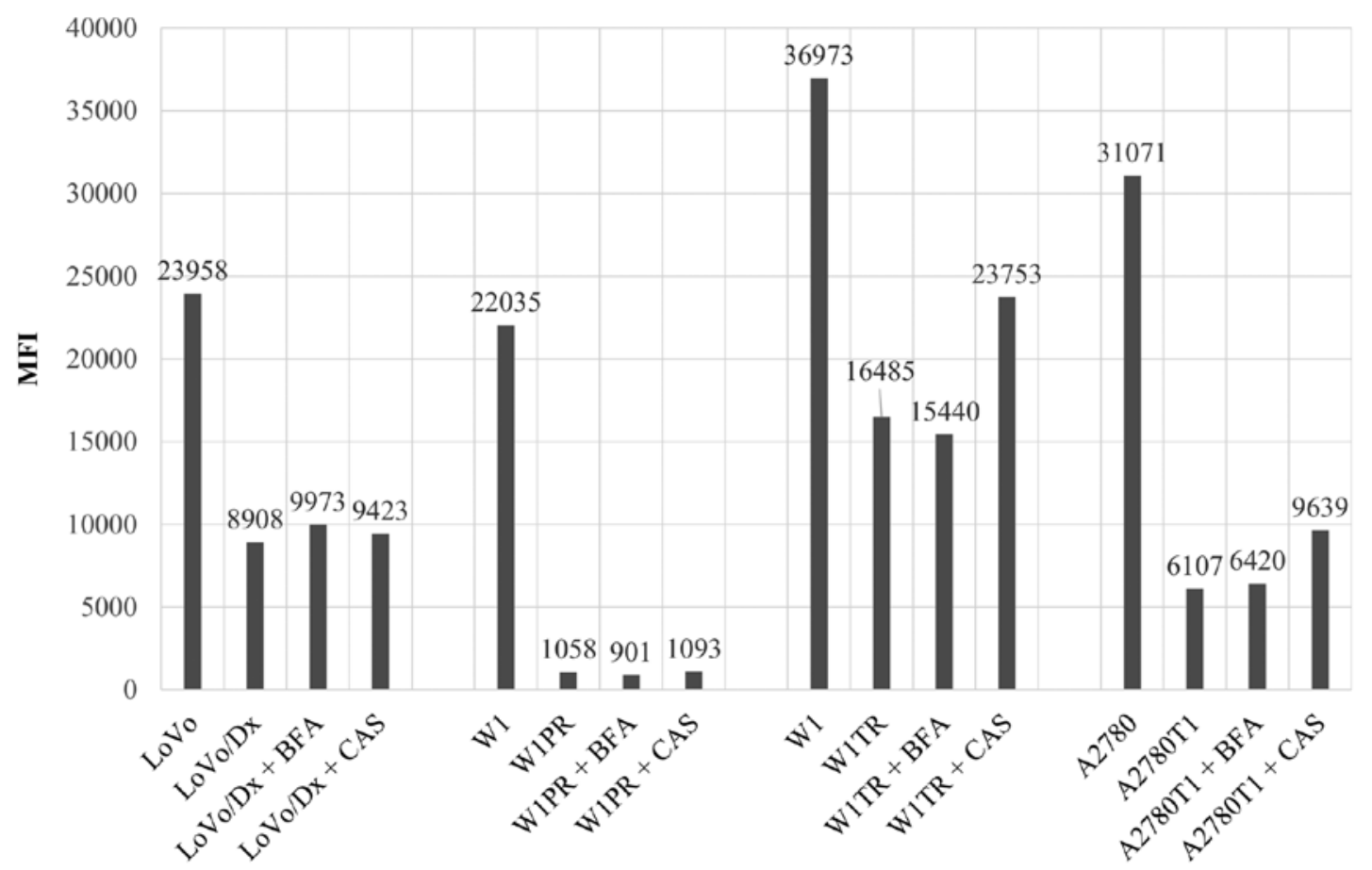

Figure 6. Mean fluorescence intensity (MIF) of Rho123 in LoVo, LoVo/Dx, W1 and W1PR cells and H33342 in W1, W1TR, A2780 and A2780T1 cells and in the respective cells treated with BFA or CAS. The MFI was calculated automatically using the FACS Express Plus software program.

agree with the opinion that BFA may have a sensitizing effect, depending on the drug resistance level, mainly by reversing Pgp-mediated resistance (30).
The expression of BCRP in W1TR and A2780TR1 cell lines is responsible for top resistance. We did not observe any significant changes in top resistance following BFA 
co-treatment in these cell lines. It has been reported that BFA has both a sensitizing and a protective effect, depending on the drug resistance level (30). This finding could explain why we did not notice any significant changes in W1TR and A2780T1 cell viability during co-treatment with BFA and drugs. The BFA concentrations used in this experiments may not have been sufficient for reversal of the BCRP resistant effect. Wlodkowic et al showed that BFA had a different effect on cell proliferation in various human follicular lymphoma cell lines (28). We suspect that ovarian cancer cell lines may not be susceptible to BFA.

The results of the immunoblot analysis demonstrated that BFA partially blocked the $N$-linked glycosylation of Pgp in the W1PR and LoVo/Dx cell lines. We observed bands that were approximately a few $\mathrm{kDa}$ smaller than the control bands, but they were not characteristic of Pgp lacking sugar as determined by treatment with PNGase F. It is possible that the concentration of BFA used in the present study was insufficient to completely inhibit Pgp glycosylation. BFA has not been used as an anticancer drug to date, but it has been shown to induce p53-independent apoptosis in human cancer cells $(25,29,47)$, and internucleosomal DNA fragmentation associated with apoptosis (25). Thus, we assessed the level of p53 expression in all of the tested cell lines. Our results were inconsistent with some of the literature since we did not observe any differences in p53 expression in cells that were treated with BFA but rather in the W1TR cell line, which displayed lower p53 expression. An explanation for this phenomenon is provided by Lee $e t$ al, who showed that BFA caused concentration-dependent changes in apoptosis-related protein levels and that these effects were also linked to an increase in p53 levels (26). The discrepancy in 553 expression may be a consequence of the association of apoptosis induced by BFA and mitochondrial breaching and caspases activation $(27,28)$. Thus, we evaluated the expression of CASP 3 in BFA-treated cells. All of the control cells without BFA possessed the protein, which is consistent with the previous supplementation of the cells with cytostatic drugs that could initiate the apoptotic cascade. In BFA-treated W1TR cells, we observed elevated CASP 3 expression, demonstrating that in this example, BFA potently induced apoptosis associated with caspase activation. This finding is in agreement with a previous report and confirms the involvement of caspase in cell death in this model (28). In contrast, in W1PR cells treated with BFA, a lower level of CASP 3 expression was observed, revealing that in various cell lines BFA can affect other signaling pathways. Western blot analysis was also used to detect BCRP protein in the A2780T1 and W1TR cell lines before and after BFA supplementation. In the control samples, two bands were identified that were consistent with antibody specifications. These two bands represented the unglycosylated and fully glycosylated forms of BCRP. In the BFA-treated cells, in addition to these two bands, we observed another band corresponding to a protein with a smaller molecular weight. Nakagawa et al also identified additional bands in Flip-In-293/ABCG2 cells that had been incubated with BFA and suggested that they were unglycosylated or immature forms of BCRP (15). We suggest that BFA caused the partial degradation of BCRP, probably due to ERAD in which misfolded proteins are retrotranslocated from the ER to the cytosol and subsequently degraded by the ubiquitin-proteasome system $(16,22)$. We demonstrated that $N$-linked glycosylation was essential for BCRP expression and that the disruption of this process resulted in protein expression, confirming a previous study (14). To examine the potential impact of BFA on BCRP protein degradation in both cell lines, we used two inhibitors of protein degradation, BMA and MG132. However, in the presence of these inhibitors, the degraded BCRP was barely visible, which may indicate that BFA did not result in proteasomal or lysosomal degradation alone but that a more complicated pathway could be involved, as claimed by Ferris et al (16).

In LoVo/Dx and W1PR cells treated with BFA, we did not observe any changes in the localization of the immunofluorescent Pgp signal in comparison to the control without drugs. However, Labroille et al showed a $35 \%$ decrease in the level of surface Pgp when resistant cells were treated for $24 \mathrm{~h}$ with BFA (48). Similarly, Fu et al showed that $5 \mu \mathrm{g} / \mathrm{ml}$ BFA completely blocked intracellular trafficking of Pgp to the cell surface in HeLa cells (41). In the present study, a similar phenomenon was not detected through $72 \mathrm{~h}$ of incubation with BFA. We suspect that the inhibition of BFA was not completely efficient, and therefore Pgp was still observed in the cell membrane in the W1PR cell line. The time needed to transport Pgp from the ER to the Golgi and finally to the plasma membrane is $12-48 \mathrm{~h}$ (41), and therefore our 72-h incubation should have been sufficient. However, another study showed that the total level of Pgp was stable in BFA-treated K562 MDR cells (48), indicating that the surface Pgp pool is continuously supplied by the cytoplasmic pool via the migration of Golgi vesicles containing Pgp towards the cell surface (48). Immunofluorescent analysis of Pgp revealed granules in W1PR cells treated with BFA and similar granules with positive staining for BCRP in the A2780T1 cell line. These findings could be explained by a higher condensation of Pgp/BCRP in the cytoplasm induced by BFA disorders in the intercellular protein trafficking of newly secreted proteins. Furthermore, in the BFA-treated W1PR cells, we observed an accumulation of Pgp close to the nucleus. Our explanation is consistent with that of other researchers. The entrapment of insoluble forms of a protein in the ER is commonly observed (16). Granules can be describe as a trapped, newly synthesized Pgp in the fused Golgi-ER compartment (41) since BFA could cause the collapse of Golgi membranes into the ER (47). The accumulation of protein may be associated with an inefficient detection of misfolded protein (19) induced by BFA treatment, or it could indicate that the protein was degraded through ERAD (16). BFA did not have a significant effect on Pgp expression in LoVo/Dx cells, probably due to the large intracellular pool of Pgp. We observed a clear change only in the morphology of the cells, which become rounder. A similar effect was observed for BCRP in the W1TR cell line after BFA incubation. This finding may indicate that the concentration of BFA was slightly toxic to the cells. However, we did not observe nuclear condensation or fragmentation, which is characteristic of apoptotic cells $(26,28)$. The results may have been caused by the ability of BFA to block cell adhesion, as demonstrated in the GR and OVCAR-3 cell lines $(24,26)$. BFA started to induce apoptosis associated with mitochondrial breaching and caspase activation, as demonstrated in human follicular lymphoma cell lines (28). We confirmed that the morpholog- 
ical changes induced in BFA-exposed cells could be dependent on cell cycle arrest (24).

CAS is an $\alpha$-glucosidase inhibitor. During the simultaneous treatment with CAS and drugs, we observed a decrease in cell viability, but this change was not statistically significant. This result could indicate that CAS did not significantly affect the activity of glycoproteins. Nakajima et al also showed that CAS treatment had an insignificant effect on UGTA9 activity in HEK293 cells (17). In contrast to our results, a smaller reversal effect of CAS on cellular resistance has been shown in Flip-In-293/ABCG2 cells (15). It has also been demonstrated that CAS reduced the proliferative properties of $\mathrm{C} 6$ glioblastoma cells (37). Its analogues SO-OCS and CO-OCS reduced cell proliferation by inducing cell cycle arrest in breast cancer cell lines and induced cell death in cancer cells via $B c l 2$ family proteins (49). The discrepancies between the literature and our results may be explained by differential effects of CAS on different cell types.

The results of the western blot analysis showed that CAS had no effect on Pgp expression. In control samples of BCRP using the A2780T1 cell line, two bands were observed, which was similar to the results obtained with BFA. These two bands represented the unglycosylated and fully glycosylated forms of BCRP. In the W1TR cells, we noted only one, the heavier band, suggesting that this sample contained mainly one form of BCRP, the fully glycosylated form. In both cases, in the A2780T1 and W1TR cell lines, CAS treatment resulted principally in the appearance of the lower bands. In our opinion, this result demonstrated that CAS blocked $\mathrm{N}$-glycosylation of the BCRP protein. In contrast, Nakagawa et al showed that incubation with CAS only reduced the level of BCRP protein expression in Flip-In-293/ABCG2 cells, but no additional bands with a lower molecular weight were observed (15). Notably, the level of p53 expression was reduced in all of the cells that were supplemented with CAS. A few possible explanations may explain the reduced expression of p53. One explanation, which has been described in HL-60 cells, is that the gene was deleted or mutations occurred in the p53 promoter or within regulatory regions (50). It has been well established that the loss of p53 prevents cellular senescence and apoptosis (51), which likely explains why we were unable to detect a decrease in cell viability in response to CAS treatment.

We wanted to determine whether CAS had any influence on the cellular localization of MDR proteins. The immunofluorescence results revealed that the Pgp signal around the nucleus was diminished in cells treated with CAS. Furthermore, in the LoVo/Dx cells, we observed a granular signal in the cytoplasm. Similarly, a granular and tubular signal was observed for BCRP in the A2780T1 cells. These results indicated that CAS did not significantly modify the export of surface glycoproteins and that it could abrogate protein transport in the cytoplasm. Lin et al showed that CAS may lead to an accumulation of unfolded proteins in the ER due to improper glycosylation (52). We propose a similar explanation for the effects of CAS treatment in the present study. We also suggest, in agreement with a previous report, that the disruption of BCRP $N$-linked glycosylation may enhance ubiquitin-mediated proteasomal degradation (15). The $N$-linked glycan of BCRP could be a checkpoint, and it has been considered to be important for the stabilization of nascent BCRP protein (15). We did not observe any changes in W1TR cells supplemented with CAS. Schraen-Maschke and Zanetta also showed that C6 glioblastoma cells treated with CAS displayed reduced re-aggregation (37).

We assessed whether BFA or CAS increases the intercellular accumulation of drugs in resistant cell lines. We showed that whole, sensitive versions of cancer cell lines retained a greater amount of fluorescent dye in comparison with the resistant cell lines. Similarly, Meschini et al observed high levels of dox in LoVo cells and only very low amounts of drug in LoVo/ Dx cells (6). Furthermore, Verovski et al showed significantly reduced Rho1 23 uptake in Pgp-positive H134AD cells relative to H134 cells (30). These findings confirmed the hypothesis that resistant cell lines express MDR proteins that protect the cells against the cytotoxic effects of drugs. For this reason, the MFI values for Rho123 or H33342 were reduced in resistant cells. We noted that BFA increased the accumulation of fluorescent dye only in the $\mathrm{LoVo} / \mathrm{Dx}$ cells. This result confirmed our findings using the MTT test, in which BFA caused the most statistically significant decrease in cell viability in $\mathrm{LoVo} / \mathrm{Dx}$ cells. Janneh et al also showed that BFA increased the accumulation of zidovudine in 3T3-F442A pre-adipocytes (42). We agree with the finding that BFA had a toxic effect mainly toward $\mathrm{LoV} / \mathrm{Dx}$ cells that was similar to the effect reported for OVCAR-3 cells (26). In W1TR cells, BFA had a small protective effect (decrease in MFI). Similar results have been described by Verovski et al, who showed a decrease in cellular dox content in PSN1/ADR cells that were treated with BFA (30). It is possible that BFA plays a role in drug resistance associated with the Pgp transporter, but only in selected types of cancers. Flow cytometric analyses showed that CAS had no effect on the accumulation of Rho123 in W1PR cells, but in the remaining cell lines, it was very important for pump activity. We detected the greatest increase in $\mathrm{H} 33342$ accumulation in W1TR cells, a somewhat smaller increase in A2780T1 cells and the smallest increase in LoVo/Dx cells. These results differ from those obtained for the MTT assay. Although the cell viability did not decrease in response to CAS administration, an increased accumulation of drug was observed by flow cytometry. In our opinion, this finding indicates that the pumps work in the presence of CAS but function at a somewhat slower rate. As a result, using flow cytometry, we observed an increased concentration of drug shortly after exposure of the cells to the dyes. These results are difficult to discuss since similar experiments with CAS have not been reported.

These results suggest that BFA could be a candidate anticancer drug supplement, mainly in cells that are characterized by Pgp overexpression. CAS has therapeutic potential, but it must be evaluated in other cell lines. These compounds are likely to be useful as supplements in anticancer therapy.

\section{Acknowledgements}

The present was supported by the Poznan University of Medical Sciences 502-14-02229373-10079 to K.W.

\section{References}

1. Christiansen MN, Chik J, Lee L, Anugraham M, Abrahams JL and Packer NH: Cell surface protein glycosylation in cancer. Proteomics 14: 525-546, 2014. 
2. Persidis A: Cancer multidrug resistance. Nat Biotechnol 18 (Suppl): IT18-IT20, 2000.

3. Sharom FJ: ABC multidrug transporters: Structure, function and role in chemoresistance. Pharmacogenomics 9: 105-127, 2008.

4. Ma H, Miao X, Ma Q, Zheng W, Zhou H and Jia L: Functional roles of glycogene and $\mathrm{N}$-glycan in multidrug resistance of human breast cancer cells. IUBMB Life 65: 409-422, 2013.

5. Li K, Sun Z, Zheng J, Lu Y, Bian Y, Ye M, Wang X, Nie Y, Zou H and Fan D: In-depth research of multidrug resistance related cell surface glycoproteome in gastric cancer. J Proteomics 82: 130-140, 2013.

6. Meschini S, Calcabrini A, Monti E, Del Bufalo D, Stringaro A, Dolfini E and Arancia G: Intracellular P-glycoprotein expression is associated with the intrinsic multidrug resistance phenotype in human colon adenocarcinoma cells. Int J Cancer 87: 615-628, 2000.

7. Pastan I and Gottesman M: Multiple-drug resistance in human cancer. N Engl J Med 316: 1388-1393, 1987.

8. Wojtowicz K, Szaflarski W, Januchowski R, Zawierucha P, Nowicki $\mathrm{M}$ and Zabel M: Inhibitors of $\mathrm{N}$-glycosylation as a potential tool for analysis of the mechanism of action and cellular localisation of glycoprotein P. Acta Biochim Pol 59: 445-450, 2012.

9. Shapiro AB, Fox K, Lee P, Yang YD and Ling V: Functional intracellular P-glycoprotein. Int J Cancer 76: 857-864, 1998.

10. Baldini N, Scotlandi K, Serra M, Shikita T, Zini N, Ognibene A, Santi S, Ferracini R and Maraldi NM: Nuclear immunolocalization of P-glycoprotein in multidrug-resistant cell lines showing similar mechanisms of doxorubicin distribution. Eur J Cell Biol 68: 226-239, 1995.

11. Calcabrini A, Meschini S, Stringaro A, Cianfriglia M, Arancia G and Molinari A: Detection of P-glycoprotein in the nuclear envelope of multidrug resistant cells. Histochem J 32: 599-606, 2000 .

12. Noguchi K, Katayama K and Sugimoto Y: Human ABC transporter ABCG2/BCRP expression in chemoresistance: Basic and clinical perspectives for molecular cancer therapeutics. Pharm Genomics Pers Med 7: 53-64, 2014.

13. Nakanishi $T$ and Ross DD: Breast cancer resistance protein (BCRP/ABCG2): Its role in multidrug resistance and regulation of its gene expression. Chin J Cancer 31: 73-99, 2012.

14. Wakabayashi-Nakao K, Tamura A, Furukawa T, Nakagawa $H$ and Ishikawa T: Quality control of human ABCG2 protein in the endoplasmic reticulum: Ubiquitination and proteasomal degradation. Adv Drug Deliv Rev 61: 66-72, 2009.

15. Nakagawa H, Wakabayashi-Nakao K, Tamura A, Toyoda Y, Koshiba S and Ishikawa T: Disruption of N-linked glycosylation enhances ubiquitin-mediated proteasomal degradation of the human ATP-binding cassette transporter ABCG2. FEBS J 276 7237-7252, 2009.

16. Ferris SP, Kodali VK and Kaufman RJ: Glycoprotein folding and quality-control mechanisms in protein-folding diseases. Dis Model Mech 7: 331-341, 2014.

17. Nakajima M, Koga T, Sakai H, Yamanaka H, Fujiwara R and Yokoi T: $N$-Glycosylation plays a role in protein folding of human UGT1A9. Biochem Pharmacol 79: 1165-1172, 2010.

18. Tannous A, Pisoni GB, Hebert DN and Molinari M: N-linked sugar-regulated protein folding and quality control in the ER. Semin Cell Dev Biol 41: 79-89, 2015

19. Ruggiano A, Foresti O and Carvalho P: Quality control ER-associated degradation: protein quality control and beyond. J Cell Biol 204: 869-879, 2014.

20. Klausner RD, Donaldson JG and Lippincott-Schwartz J: Brefeldin A: Insights into the control of membrane traffic and organelle structure. J Cell Biol 116: 1071-1080, 1992.

21. Oda K, Fujiwara T and Ikehara Y: Brefeldin A arrests the intracellular transport of viral envelope proteins in primary cultured rat hepatocytes and HepG2 cells. Biochem J 265: 161-167, 1990.

22. Misumi Y, Misumi Y, Miki K, Takatsuki A, Tamura G and Ikehara Y: Novel blockade by brefeldin A of intracellular transport of secretory proteins in cultured rat hepatocytes. J Biol Chem 261: 11398-11403, 1986.

23. Yardin C, Terro F, Esclaire F, Rigaud M and Hugon J: Brefeldin A-induced apoptosis is expressed in rat neurons with dephosphorylated tau protein. Neurosci Lett 250: 1-4, 1998.

24. Pommepuy I, Terro F, Petit B, Trimoreau F, Bellet V, Robert S, Hugon J, Labrousse F and Yardin C: Brefeldin A induces apoptosis and cell cycle blockade in glioblastoma cell lines. Oncology 64: 459-467, 2003.
25. Shao RG, Shimizu T and Pommier Y: Brefeldin A is a potent inducer of apoptosis in human cancer cells independently of p53. Exp Cell Res 227: 190-196, 1996.

26. Lee SA, Kim YJ and Lee CS: Brefeldin A induces apoptosis by activating the mitochondrial and death receptor pathways and inhibits focal adhesion kinase-mediated cell invasion. Basic Clin Pharmacol Toxicol 113: 329-338, 2013

27. Salles FT, Hespanhol AM, Jaeger RG and Marques MM Brefeldin-A induces apoptosis in human adenoid cystic carcinoma cultured cells. Oral Oncol 40: 585-590, 2004

28. Wlodkowic D, Skommer J and Pelkonen J: Brefeldin A triggers apoptosis associated with mitochondrial breach and enhances HA14-1- and anti-Fas-mediated cell killing in follicular lymphoma cells. Leuk Res 31: 1687-1700, 2007.

29. Wallen E, Sellers RG and Peehl DM: Brefeldin A induces p53-independent apoptosis in primary cultures of human prostatic cancer cells. J Urol 164: 836-841, 2000

30. Verovski VN, Van den Berge DL, Delvaeye MM, Scheper RJ, De Neve WJ and Storme GA: Low-level doxorubicin resistance in P-glycoprotein-negative human pancreatic tumour PSN1/ADR cells implicates a brefeldin A-sensitive mechanism of drug extrusion. Br J Cancer 73: 596-602, 1996.

31. Hohenschutz LD, Bell EA, Jewess PJ, Leworthy DP, Pryce RJ, Arnold E and Clardy J: Castanospermine, a 1,6,7,8-tetrahydroxyoctahydroindolizine alkaloid from seeds of Castanospermum austarale. Phytochemistry 20: 811-814, 1981.

32. Pili R, Chang J, Partis RA, Mueller RA, Chrest FJ and Passaniti A: The $\alpha$-glucosidase I inhibitor castanospermine alters endothelial cell glycosylation, prevents angiogenesis, and inhibits tumor growth. Cancer Res 55: 2920-2926, 1995

33. Grochowicz PM, Hibberd AD, Bowen KM, Clark DA, Pang G, Grochowicz LK, Willenborg DO and Cowden WB: Synergism of castanospermine and FK 506. Transplant Proc 27: 355-356, 1995.

34. Vlietinck AJ, De Bruyne T, Apers S and Pieters LA: Plant-derived leading compounds for chemotherapy of human immunodeficiency virus (HIV) infection. Planta Med 64: 97-109, 1998.

35. Chang J, Block TM and Guo JT: Antiviral therapies targeting host ER alpha-glucosidases: Current status and future directions. Antiviral Res 99: 251-260, 2013.

36. Kato A, Hirokami Y, Kinami K, Tsuji Y, Miyawaki S, Adachi I, Hollinshead J, Nash RJ, Kiappes JL, Zitzmann N, et al: Isolation and SAR studies of bicyclic iminosugars from Castanospermum australe as glycosidase inhibitors. Phytochemistry 111: 124-131, 2015.

37. Schraen-Maschke S and Zanetta JP: Role of oligomannosidic $\mathrm{N}$-glycans in the proliferation, adhesion and signalling of C6 glioblastoma cells. Biochimie 85: 219-229, 2003.

38. Wojtowicz K, Januchowski R, Nowicki M and Zabel M: Inhibition of protein glycosylation reverses the MDR phenotype of cancer cell lines. Biomed Pharmacother 74: 49-56, 2015.

39. Januchowski R, Wojtowicz K, Sujka-Kordowska P, Andrzejewska $\mathrm{M}$ and Zabel M: MDR gene expression analysis of six drug-resistant ovarian cancer cell lines. BioMed Res Int 2013: 241763, 2013

40. Szaflarski W, Sujka-Kordowska P, Januchowski R, Wojtowicz K, Andrzejewska M, Nowicki M and Zabel M: Nuclear localization of $\mathrm{P}$-glycoprotein is responsible for protection of the nucleus from doxorubicin in the resistant LoVo cell line. Biomed Pharmacother 67: 497-502, 2013.

41. Fu D, Bebawy M, Kable EP and Roufogalis BD: Dynamic and intracellular trafficking of P-glycoprotein-EGFP fusion protein: Implications in multidrug resistance in cancer. Int J Cancer 109: 174-181, 2004.

42. Janneh O, Owen A, Bray PG, Back DJ and Pirmohamed M: The accumulation and metabolism of zidovudine in 3T3-F442A preadipocytes. Br J Pharmacol 159: 484-493, 2010.

43. Rhodes T, Barrand MA and Twentyman PR: Modification by brefeldin A, bafilomycin A1 and 7-chloro-4-nitrobenz-2-oxa1,3-diazole (NBD) of cellular accumulation and intracellular distribution of anthracyclines in the non-P-glycoprotein-mediated multidrug-resistant cell line COR-L23/R. Br J Cancer 70: 60-66, 1994.

44. Wood DJT, Rumsby MG and Warr JR: Monensin and verapamil do not alter intracellular localisation of daunorubicin in multidrug resistant human KB cells. Cancer Lett 108: 41-47, 1996.

45. Marquardt D and Center MS: Drug transport mechanisms in HL60 cells isolated for resistance to adriamycin: Evidence for nuclear drug accumulation and redistribution in resistant cells. Cancer Res 52: 3157-3163, 1992. 
46. Gong Y, Wang Y, Chen F, Han J, Miao J, Shao N, Fang Z and $\mathrm{Ou}$ Yang R: Identification of the subcellular localization of daunorubicin in multidrug-resistant K562 cell line. Leuk Res 24: 769-774, 2000.

47. Togawa A, Ito H, Kimura F, Shimizu H, Ohtsuka M, Shimamura F Yoshidome H, Katoh A and Miyazaki M: Establishment of gemcitabine-resistant human pancreatic cancer cells and effect of brefeldin-A on the resistant cell line. Pancreas 27: 220-224, 2003.

48. Labroille G, Belloc F, Bilhou-Nabera C, Bonnefille S, Bascans E, Boisseau MR, Bernard P and Lacombe F: Cytometric study of intracellular P-gp expression and reversal of drug resistance. Cytometry 32: 86-94, 1998.

49. Allan G, Ouadid-Ahidouch H, Sanchez-Fernandez EM, Risquez-Cuadro RÃ, Fernandez JÃMG, Ortiz-Mellet C and Ahidouch A: New castanospermine glycoside analogues inhibit breast cancer cell proliferation and induce apoptosis without affecting normal cells. PLoS One 8: e76411, 2013.
50. Durland-Busbice $S$ and Reisman D: Lack of p53 expression in human myeloid leukemias is not due to mutations in transcriptional regulatory regions of the gene. Leukemia 16: 2165-2167, 2002.

51. Muller PAJ, Vousden KH and Norman JC: p53 and its mutants in tumor cell migration and invasion. J Cell Biol 192: 209-218, 2011.

52. Lin ZP, Boller YC, Amer SM, Russell RL, Pacelli KA, Patierno SR and Kennedy KA: Prevention of brefeldin A-induced resistance to teniposide by the proteasome inhibitor MG-132: Involvement of NF-kappaB activation in drug resistance. Cancer Res 58: 3059-3065, 1998 